Gabriel MoReno GonZÁLEz, Estabilidad presupuestaria y constitución: fundamentos teóricos y aplicación desde la Unión Europea, Editorial Tirant lo Blanch, Valencia, 2019, 468 pp., ISBN 978841313963030 .

\author{
http://dx.doi.org/10.18543/ed-67(2)-2019pp407-413
}

La estabilidad presupuestaria surge como uno de los elementos más controvertidos en cuanto a su incorporación a los textos constitucionales, teniendo en cuenta los avances del Estado social, debido a las limitaciones que ello implica. Ahondando en este problema, nos encontramos actualmente en un contexto de supranacionalización de la toma de decisiones respecto de un amplio abanico de ámbitos, pues la Unión Europea ha ido recabando competencias, paulatinamente, mediante el proceso de integración que comenzó como un pacto económico-arancelario entre seis Estados, pero que, finalmente, ha desembocado en la creación de una entidad sui generis de compleja naturaleza jurídica y política.

Precisamente, en este contexto de desarrollo de la integración, es donde surge la necesidad de analizar el papel de la estabilidad presupuestaria en la Unión Europea, así como su eventual incorporación a los textos constitucionales de los Estados miembros, y es aquí donde el profesor de la Universidad de Extremadura y autor del libro que ahora recensionamos, Gabriel
Moreno, contribuye con un avance muy relevante, pues realiza con gran maestría un análisis holístico del recorrido, desarrollo e implicaciones de este concepto, la estabilidad presupuestaria, culminándolo con un detallado análisis de la importancia de los distintos mecanismos que la Unión Europea (aunque sea de forma ajena a ella) ha ido creando en pos de la juridificación de la estabilidad presupuestaria a nivel comunitario.

En la primera parte, el autor desarrolla el recorrido del concepto de estabilidad presupuestaria, desde su florecimiento, gestado en el seno de la teoría ordoliberal y del Coloquio Lippman, hasta su cristalización en el Derecho de la Unión Europea. Para ello, comienza analizando la reconstrucción del liberalismo realizada por el coloquio mentado, donde surge el neoliberalismo como ruptura de las teorías del laissez faire, de modo que ahora el Estado pasa a ser considerado un código de circulación que dicta normas generales, es decir, como un elemento activo por y para la 
competencia. En este contexto, aprovecha para analizar el ordoliberalismo surgido como superación de la concepción iusaturalista, pues el marco jurídico, cuanto más perfeccionado, hace disminuir la necesidad de adoptar medidas concretas para el desarrollo del mercado. Así, la Economía Social de Mercado se constituye, como bien dice el autor, como la manifestación concreta del neoliberalismo iniciado con el giro conceptual, copernicano, del Coloquio Lippman. La forma de conseguir estos objetivos pasa por la elevación a la indisponibilidad de una serie de apriorismos, entre ellos el de estabilidad presupuestaria, que quedarían al margen de cualquier decisión política ordinaria.

Por otra parte, encontramos también el análisis de las teorías de James M. Buchanan y del recorrido de la estabilidad presupuestaria en los Estados Unidos. Entendiendo el mercado como orden constitucional, Buchanan se refiere a la Economía Constitucional como sucesora del ordoliberalismo, pero con el matiz de la constitucionalización del marco jurídico, como medio para evitar los excesos derivados del poder de intervención estatal, de modo que se debería crear ex ante un entramado jurídico suficiente para restringir la discrecionalidad política en materia económica. Asimismo, para que la estabilidad presupuestaria constituya un límite efectivo deben incorporarse mecanismos específicos eficaces, como los referidos por el autor: los límites expresos al déficit y a la deuda pública, el federalismo fiscal como bálsamo al jacobinismo recaudatorio y los mecanismos de democracia semidirecta en la aprobación de las bases impositivas.

Las críticas a esta teoría vienen de la mano del contractualismo de Rawls, así como también resulta bastante inverosímil, por la propia naturaleza del ser humano, la afirmación de Buchanan de que el pacto debe ser lo más inclusivo posible y estar basado en la unanimidad, teniendo en cuenta, además, la actual configuración de los (cortos) mandatos políticos que no permiten a los dirigentes centrarse en objetivos a largo plazo.

Asimismo, nos llama la atención la consideración de la constitución normativa como elemento integrador de las tradiciones liberal y democrática, que a pesar de ser antagónicas en ciertos puntos, son complementarias; pero no se trata de una integración neutra, sino que una de ellas sale victoriosa el elemento liberal, surgiendo en la Economía Constitucional de Buchanan la más perfeccionada (e indisponible) vía de restricción de la voluntad de las mayorías.

Por último, en esta primera parte el autor analiza el recorrido práctico de la Golden Rule (Balanced Budget Amendment) en los Estados Unidos, donde se encontraba como una práctica consolidada, pero que carecía del marco constitucional necesario. A partir de 1970 y tras estar silenciada desde el New Deal, vuelve a situarse en el panorama político su constitucionalización. Entre las dos vías posibles, resultó elegida (por cuestiones políticas) la convocatoria de una convención constituyente que, a pesar de fracasar por no reunir el quórum requerido y debido a los avatares políti$\cos$ que el autor recoge en este 
apartado, sigue abierta debido a que muchos Estados aún no la han revocado expresamente.

En cuanto a la problemática constitucional inherente a la $B B R$, surgen primero los problemas de concreción numérica, distinguiendo entre métodos de cálculo de cash y de accrual (de caja y devengo), así como la eventual necesidad de un órgano supervisor; la de establecer suspensiones de la regla por motivos temporales (de acomodación del ordenamiento) o causales (por circunstancias excepcionales); así como la pertinencia de establecer previsiones complementarias y el alcance del ámbito institucional; o la existencia de instrumentos de evasión. Finalmente, el autor dedica un extenso análisis a la problemática de la ejecutoriedad, como posible conflicto para la división de poderes, de modo que acaba por reafirmarse en la imposibilidad de que el poder judicial decida, a través de sus sentencias, el control directo de la estabilidad presupuestaria y, por ende, de elementos esenciales del orden económico.

$$
* \quad * \quad *
$$

En la segunda parte, Gabriel Moreno enfoca su análisis en la juridificación de la estabilidad presupuestaria a nivel de la Unión Europea, así como a veces fuera de ella; y para ello, divide su contenido en tres apartados distintos, correspondientes a la creación de los tres instrumentos que ocuparán un papel protagonista en dicho proceso, como son el Pacto de Estabilidad y Crecimiento (PEC), el Mecanismo Europeo de Estabilidad (MEDE) y el Tratado de Estabilidad, Coordinación y Gobernanza de la Unión Económica y Monetaria (TECG). A la combinación de los tres el autor la denomina «tríada de mecanismos», como si de un tríptico se tratara. En consecuencia, trataremos de recoger los puntos más relevantes que el profesor ha señalado respecto de cada uno de ellos, a modo de pinceladas que sirvan de anticipo para el detallado análisis realizado en la obra.

La estabilidad presupuestaria comenzó reptando gracias a la serpiente monetaria, pero no fue hasta el Tratado de Maastricht cuando quedó incorporado al Derecho originario. El primero de los mecanismos que se creó fue el PEC, alejado de las propuestas originarias de Waigel, que constituye un sistema sancionador externo a la UE y con regulaciones de Derecho derivado, pero que acabó siendo ineficaz, como bien demuestran los incumplimientos sin sanción por parte de Alemania y Francia, que lejos de provocar una revisión del mecanismo (para aumentar su efectividad) desencadenó su flexibilización.

Asimismo, debido a que en numerosas ocasiones la estabilidad presupuestaria se trata de incorporar por la vía política, sorteando el marco jurídico de la UE, se produjeron una serie de resistencias, tanto comunitarias (por parte del TJUE, con la Sentencia del Pleno de 13 de julio de 2004), como constitucionales (por parte de los Tribunales Constitucionales nacionales, encabezados por el Alemán, con sentencias como las de Maastricht).

Sin embargo, tras su creación comenzó la crisis de los rescates en la Unión Europea, comenzando por el caso griego, de modo que el PEC y las innovaciones incorporadas por el 
Tratado de Lisboa devinieron insuficientes y surgió la necesidad de tomar nuevas medidas, como el refuerzo del PEC mediante el Derecho derivado (six y two pack) o la creación del MEDE y el TECG. El autor distingue las novedades que conlleva el six pack, principalmente respecto de las vertientes preventiva (presentación anticipada de los planes a largo plazo ante Bruselas y coordinación de los diversos procedimientos y actos del Pacto en el interior del Semestre Europeo) y correctiva (mayor protagonismo de la Comisión, así como la introducción de la más restrictiva mayoría cualificada inversa después de haberse abierto el PDE). Asimismo, señala las altas dosis de discrecionalidad en manos de la comisión por el Procedimiento de Desequilibrios Macroeconómicos (PDM). Así como, más adelante, indica que la principal novedad del two pack es la supervisión directa de los proyectos de presupuestos nacionales.

Pasamos ahora al segundo capítulo, donde estudia el MEDE como mecanismo exógeno a la UE, justificado por la excesiva rigidez del marco comunitario y la cláusula del no bailout. Teniendo en cuenta que la única posibilidad de salvar la coyuntura financiera pasaba por la vía del artículo 122.2 TFUE, surgieron los acuerdos de asistencia financiera, materializados en los Memorandum of Understanding $(\mathrm{MoU})$ celebrados entre la Comisión y el Estado en concreto; fue considerada insuficiente, se creó la FEEF, donde nos llama la atención la participación del FMI, institución ajena a la Unión Europea, acertadamente señalada por el autor como la primera muestra de la huida comunitaria y la gestación del nuevo método comunitario.

Esta FEEF fue sustituida más tarde, con la firma del MEDE, que se crea como mecanismo externo al marco comunitario y, por tanto, nace ya con el cuestionamiento de su legitimidad. Por ello, el MEDE fue enjuiciado por el TJUE en el asunto Pringle, de 27 de noviembre de 2012, donde se validó la operación y se impusieron, a su vez, dos límites, como son la compatibilidad con el Derecho originario y la interdicción del menoscabo de la esencia de las principales instituciones comunitarias (a pesar de la intervención de la Comisión). En consecuencia, el MEDE no es parte de la UE y las decisiones e instrumentos que adopte serán propios $\mathrm{y}$, por tanto, no enjuiciables bajo los parámetros de la CDFUE, pese a que esta tiene el mismo status que los Tratados constitutivos. En consecuencia, la única posibilidad de control pasa por la conformidad del $M o U$ con la coordinación de las políticas económicas de la UE y la estabilidad presupuestaria.

Precisamente, por las dudas surgidas respecto de su legitimidad, el autor analiza con mucho tino las resistencias constitucionales por parte de los Estados miembros. En primer lugar, se refiere a las resistencias al MEDE como institución, reflejando lo establecido por el TCA, donde se enjuició la compatibilidad con la Ley Fundamental de Bonn y se impusieron dos condicionantes, con el MEDE ya en vigor, por lo que se tuvo que adoptar una Declaración interpretativa para salvar esta situación; así como lo reflejado por Estonia, donde el 
Tribunal se remitió a la legitimación democrática de la adhesión. En segundo lugar, hace referencia a las resistencias relativas a la condicionalidad de los MoU asociada a la estabilidad presupuestaria, por parte de los Tribunales Constitucionales de Letonia (donde se acabaron por renegociar los términos del $M o U$ ), de Portugal (donde se dictaminó la inconstitucionalidad sin efectos, al amparo del artículo 282.4 de su Constitución) y de Grecia.

En el tercer y último capítulo, analiza el TECG como la más perfeccionada de las huidas comunitarias, negociado de forma rápida y relativamente transparente, pero sin la intervención del Parlamento Europeo. Como elementos más relevantes el autor señala su sometimiento al Derecho de la UE (efecto boomerang), su carácter abierto hacia el futuro, permitiendo la firma de aquellos que se habían negado en un principio, y la transposición en plazo máximo de cinco años a los ordenamientos nacionales. Además, pone énfasis en dos claves, como son el pacto fiscal (equilibrio) y la constitucionalización (preferente) de la estabilidad presupuestaria, que ha dado lugar a la constitucionalización flexible bendecida por Bruselas, de modo que actualmente existen supuestos de constitucionalización fuerte (Alemania, España e Italia), de falta de constitucionalización por la concepción monista del Derecho Internacional (Francia) y de constitucionalización débil (Portugal).

A pesar de las recomendaciones de utilizar la vía del artículo 126.14 TFUE, se tuvo que descartar por las negativas de Reino Unido y Chequia, pero de igual modo se descartaron otras tres opciones: la cláusula de imprevisión, la posibilidad de reforma simplificada de los tratados y la vía de la cooperación reforzada. Sin embargo, la elección de esta vía, como acertadamente afirma el autor, es una opción consciente de los Estados miembros de la Unión Europea, pero es ahí donde nos invita a reflexionar si se trata de una opción deliberada para evitar el encorsetamiento del marco comunitario y la intervención del Parlamento Europeo, o bien se trata de una elección medial, esto es, se produce la huida de dicho marco para alcanzar un telos superior, como es el beneficio de la Unión Europea. En cualquier caso, la prevista incorporación del TECG al acervo comunitario supondría mejoras en cuanto al control y la legitimidad de la opción elegida.

En cuanto a la problemática de la transposición, se señalan dos paradojas: en primer lugar, respecto del plazo de un año, impracticable en muchos casos por la propia configuración constitucional nacional; $y$, en segundo lugar, respecto de la permanencia del marco normativo, impulsado por el país germano, como bien se deduce de las declaraciones de Merkel, que acaba por convertirse en una declaración simbólica. En una primera aproximación, podría resultar extraño que Alemania, donde se ha gestado la construcción de la identidad nacional como contrapeso al Derecho de la UE, no se haya opuesto a este proceso, pero señala Gabriel Moreno de forma brillante que la estabilidad presupuestaria es una regla constitucionalizada en la LFB, de modo que no es una 
afrenta a su constitucionalismo, sino que es la mejor forma para extender sus parámetros al resto de Estados.

Finalmente, se recoge una amplia comparativa entre la experiencia europea y la BBR norteamericana, de modo que además de las diferencias estructurales existentes entre ambos sistemas, hace referencia a los métodos de cálculo (estructural/nominal), a las reglas temporales o de acomodación, la existencia o no de instrumentos de evasión o la ejecutoriedad judicial.

$$
* \quad * \quad *
$$

En la tercera y última parte, el autor analiza la nueva constitución económica neoliberal de la Unión Europea, subdividiendo la exposición en dos apartados: por un lado, el análisis del mercado como principio rector; $y$, por otro, la incompatibilidad de los que denomina como modelos económico competitivo y político fragmentado con la parte dogmática del Estado social. Además, de forma transversal, realiza una importante reflexión acerca del papel del TJUE a lo largo del proceso.

En el primer capítulo, comienza exponiendo el desarrollo de la integración europea, donde la competencia ha acabado por convertirse en el criterio rector, por encima de las consideraciones nacionales al respecto. En consonancia, se ha superado el rígido marco del Estado-nación, pero sin la existencia de un demos europeo que lo sostenga, pues ha sido sustituido por la competencia como sostén artificial para este entramado.

Sin embargo, la constitución económica europea no se puede identificar con la construcción ordoliberal, ya que esta última es excesivamente complicada y la interrelación entre teoría y práctica nunca es unidireccional, aunque el ordoliberalismo pueda servir como parapeto teórico para las consecuciones jurídicas del mercado y la competencia como principios estructurantes de la Unión. Asimismo, concluye que con su implantación se ha superado la antigua concepción de la Constitución como horquilla de acción para la política estatal, pues la mutación que ha sufrido con la incorporación de la estabilidad presupuestaria ha acabado por extinguir casi en su totalidad el marco de apertura al pluralismo, suponiendo, a su vez, un grave conflicto con la construcción del Estado social. Asimismo, vuelven a reavivarse las dudas sobre la legitimación, en este caso respecto del que llama nuevo «método plutocrático europeo», caracterizado por constituirse en esferas extracomunitarias, con la introducción de la regla de mayoría cualificada inversa y la ausencia de intervención del Parlamento Europeo.

Como bien entiende Gabriel Moreno, la constitución económica europea puede explicarse desde la convergencia teórica del ordoliberalismo y de la Economía Constitucional, esto es, de la elevación a la indisponibilidad de la estabilidad presupuestaria, introduciendo esta nueva concepción amplia de la Constitución y del constitucionalismo como técnica de control político. Con ella, triunfa el modelo de integración negativa (económico competitivo) y el modelo político fragmentado, pues no existe un demos común que pueda provocar una resistencia efectiva al nuevo método 
plutocrático europeo y a las esferas de indisponibilidad que ahora se crean.

En el segundo capítulo, por su parte, se refiere a la incompatibilidad de estos modelos (económico competitivo y político fragmentado) con la parte dogmática del Estado social, pues se configura la estabilidad presupuestaria como una camisa de fuerza que, como ya avanzamos, dinamita los márgenes de pluralismo que antes existían a nivel nacional, tratando de evitar que ese federalismo competitivo se convierta en una race to the bottom. Además, hace referencia a las últimas (pero insuficientes) resistencias constitucionales, de mano de la transformación de la jurisprudencia del TJUE, desde el caso Pringle (donde se bendecía la expulsión de la CDFUE como parámetro de control) hasta Ledra Advertising, donde se produce un tímido e insuficiente cambio.

Finalmente, dedica un último apartado a desgranar los frutos de su investigación, compilando las características de la nueva constitución económica neoliberal de la Unión Europea, entre los que podríamos destacar, a modo de muestra, la victoria total del elemento liberal frente al democrático en el seno de la democracia constitucional, y el consiguiente choque con el Estado social y los derechos fundamentales que de ella se derivan.

Como consecuencia de todo lo expuesto, podemos afirmar sin lugar a dudas que la lectura de la obra que ahora recensionamos es absolutamente necesaria para todo aquel que quiera comprender los cimientos sobre los que hunde sus raíces la estabilidad presupuestaria, las implicaciones que trae consigo la constitucionalización de este concepto a nivel nacional y el papel que en ello ha desempeñado la Unión Europea a través de la creación de los distintos mecanismos que tienen por objeto circunscribir el margen de acción pluralista de nuestras democracias contemporáneas.

Juan Barroso Márquez

Investigador en Derecho Constitucional Universidad de Extremadura 


\title{
Gabriel Moreno GonZÁlez, Estabilidad presupuestaria y constitu- ción: fundamentos teóricos y aplicación desde la Unión Euro- pea, Editorial Tirant lo Blanch, Valencia, 2019, 468 pp., ISBN 978841313963030.
}

\author{
http://dx.doi.org/10.18543/ed-67(2)-2019pp407-413
}

\section{Copyright}

Estudios de Deusto es una revista de acceso abierto, lo que significa que es de libre acceso en su in tegridad. Se permite su lectura, la búsqueda, descarga, distribución y reutilización legal en cualquier tipo de soporte sólo para fines no comerciales, sin la previa autorización del editor o el autor, siempre que la obra original sea debidamente citada y cualquier cambio en el original esté claramente indicado

Estudios de Deusto is an Open Access journal which means that it is free for full access, reading, search, download, distribution, and lawful reuse in any medium only for non-commercial purposes, without prior permission from the Publisher or the author; provided the original work is properly cited and any changes to the original are clearly indicated. 